Article

\title{
Characterization of Two Toxin-Antitoxin Systems in Deep-Sea Streptomyces sp. SCSIO 02999
}

\author{
Waner Zhan ${ }^{1,2}$, Jianyun Yao ${ }^{1}$, Kaihao Tang ${ }^{1}$, Yangmei $\mathrm{Li}^{1,2}$, Yunxue Guo ${ }^{1, *(\mathbb{D}) \text { and }}$ \\ Xiaoxue Wang ${ }^{1,2, *}$
}

1 Key Laboratory of Tropical Marine Bio-resources and Ecology, Guangdong Key Laboratory of Marine Materia Medica, RNAM Center for Marine Microbiology, South China Sea Institute of Oceanology, Chinese Academy of Sciences, Guangzhou 510301, China; zhanwaner16@mails.ucas.ac.cn (W.Z.); jianyunyao@scsio.ac.cn (J.Y.); khtang@scsio.ac.cn (K.T.); liyangmei13@mails.ucas.ac.cn (Y.L.)

2 University of Chinese Academy of Sciences, Beijing 100049, China

* Correspondence: yunxueguo@scsio.ac.cn (Y.G.); xxwang@scsio.ac.cn (X.W.)

Received: 22 February 2019; Accepted: 1 April 2019; Published: 4 April 2019

\begin{abstract}
Toxin-antitoxin (TA) systems are ubiquitous and abundant genetic elements in bacteria and archaea. Most previous TA studies have focused on commensal and pathogenic bacteria, but have rarely focused on marine bacteria, especially those isolated from the deep sea. Here, we identified and characterized three putative TA pairs in the deep-sea-derived Streptomyces sp. strain SCSIO 02999. Our results showed that Orf5461/Orf5462 and Orf2769/Orf2770 are bona fide TA pairs. We provide several lines of evidence to demonstrate that Orf5461 and Orf5462 constitute a type-II TA pair that are homologous to the YoeB/YefM TA pair from Escherichia coli. Although YoeB from SCSIO 02999 was toxic to an E. coli host, the homologous YefM antitoxin from SCSIO 02999 did not neutralize the toxic effect of YoeB from E. coli. For the Orf2769/Orf2770 TA pair, Orf2769 overexpression caused significant cell elongation and could lead to cell death in E. coli, and the neighboring Orf2770 could neutralize the toxic effect of Orf2769. However, no homologous toxin or antitoxin was found for this pair, and no direct interaction was found between Orf2769 and Orf2770. These results suggest that Orf2769 and Orf2770 may constitute a novel TA pair. Thus, deep-sea bacteria harbor typical and novel TA pairs. The biochemical and physiological functions of different TAs in deep-sea bacteria warrant further investigation.
\end{abstract}

Keywords: toxin-antitoxin system; YoeB/YefM; deep sea; Streptomyces

\section{Introduction}

Toxin-antitoxin (TA) systems are ubiquitous genetic elements that consist of a toxin and an antitoxin that specifically neutralize the cognate toxin [1,2]. Based on the nature of the antitoxin and the mode of interaction, TA systems have been broadly divided into six types (types I-VI) [3,4]. Previous work on TA systems has mostly focused on E. coli strains and pathogens. To date, more than 37 TA systems have been identified in E. coli K-12 MG1655 [1]. TA systems have also been identified in some major pathogens, such as Streptococcus pneumonia [5], Mycobacterium tuberculosis [6], Salmonella [7], and Pseudomonas aeruginosa [8-10]. Although most of the TAs are well studied in E. coli, TAs with new functions and characteristics have recently been discovered in other microorganisms. For example, the type-III TAs ToxI/ToxN were first characterized in the Erwinia carotovora subspecies atroseptica [11] and the toxin ToxN acts as an endoribonuclease [12]. In addition, the newly named type-VI TA SocB/SocA system was identified in Caulobacter crescentus, and the toxin SocB can interact with the sliding clamp (driving replication fork collapse) to block replication elongation [13]. The novel toxin ParS of the ParS/ParT TA pair, a mono-ADP-ribosyltransferase (mART), specifically modifies 
phosphoribosyl pyrophosphate synthetase in soil bacterium Sphingobium sp. [14]. The novel toxin DarT of the DarT/DarG TA pair from the pathogen Mycobacterium tuberculosis causes reversible DNA ADP-ribosylation [15]. Furthermore, the toxin AtaT of the AtaT/AtaR TA pair from enterohemorrhagic E. coli blocks translation initiation by $N$-acetylation of the initiator $\mathrm{tRNA}^{\mathrm{fMet}}[16,17]$. TA systems have been found to be widely distributed in microorganisms of freshwater and marine sources using bioinformatics analysis, and some of these TAs have been verified and studied. For example, seven TAs in the estuarine Synechococcus strain [18] and three TAs, including HipA/HipB, HEPN/MNT and ParEso/CopAso, in Shewanella oneidensis isolated from Lake Oneida (NY) [19-21] were identified and characterized recently. Although the marine ecosystem harbors the largest quantities and greatest diversity of microorganisms [22], the TAs in marine bacteria are much less well explored. The type-II TA pair VapC/VapB from a deep-sea Streptomyces sp. has been verified [23] and the antitoxin ToxN (cognate toxin ToxI) in the marine bacterium Vibrio harveyi [11] and toxin PndA (homologous to the toxin Hok) in the marine pathogen Vibrio parahaemolyticus [24] have been predicted. Thus, new types of TA systems or toxins with novel functions remain to be identified in extreme marine environments.

Streptomyces sp. SCSIO 02999 (hereafter referred to as SCSIO 02999) was isolated from South China Sea sediment at a depth of $880 \mathrm{~m}$ and is taxonomically close to Streptomyces sp. VTT E-062988, ACT-40 and 1A01691 [25]. SCSIO 02999 is a Gram-positive bacterium and can produce multifarious biologically active compounds with antiviral, antitumor or antibacterial activity [26-29]. We have previously characterized a type-II TA pair VapC/VapB in SCSIO 02999 [23]. In this study, we analyzed three putative TA pairs in SCSIO 02999, Orf5461/Orf5462, Orf2769/Orf2770, and Orf2767/Orf2766 and showed that Orf5461/Orf5462 and Orf2769/Orf2770 are bona fide TA pairs. We demonstrated that Orf5461/Orf5462 is homologous to the type-II YoeB/YefM TA pair in E. coli. Overexpressing YoeB from SCSIO 02999 was toxic to an E. coli host, but the antitoxin YefM from SCSIO 02999 could not neutralize the toxic effect of YoeB from E. coli. Orf2769 and Orf2770 may constitute a novel TA pair, and the overexpression of Orf2769 led to the formation of elongated E. coli cells. Further studies are needed to explore the physiological functions of different TA systems in deep-sea bacteria.

\section{Results}

\subsection{Identification of Two TA Pairs in Streptomyces sp. SCSIO 02999}

Four potential TA loci (orf3703/orf3704, orf5461/orf5462, orf2769/orf2770 and orf2767/orf2766) in the SCSIO 02999 genome have been predicted by the web-based tool RASTA-Bacteria [30] (Figure 1A). Among them, Orf3703/Orf3704 belong to the type-II TA VapC/VapB system [23]. The remaining three putative TAs were all investigated in this study. We first cloned the putative toxin gene and the TA operon for toxicity for these three pairs. Orf5461 and orf5462 encode two small proteins of 84 aa and 87 aa (Figure S1). Orf5461 belongs to the toxin YoeB family (PF06769) and Orf5462 belongs to the antitoxin YefM family (PF02604) in the Pfam database (Table S1). At the amino acid sequence level, Orf5461 shares 51\% identity with YoeB in E. coli, and Orf5462 shares 72\% identity with YefM in E. coli [31] (Figure S2A,B). Thus, we renamed this pair YoeB/YefM. As expected, overexpressing yoe $B$ via $p C A 24 \mathrm{~N}-y o e B$ is toxic, while coexpressing yoe B and yef $M$ via pCA24N-yefM-yoeB significantly reduced the toxicity of YoeB in the E. coli K12 host (Figure 1B and Figure S3A). Another putative TA pair, Orf2769 and Orf2770, encodes two small proteins of 90 aa and 83 aa (Figure S4). Overexpressing orf 2769 via pCA24N-2769 is toxic, and overexpressing orf 2770 via pCA24N-2770 showed slight growth inhibition. In contrast, coexpressing orf 2769 and orf 2770 via pCA24N-2769-2770 is not toxic in the E. coli K12 host (Figure 1C and Figure S3B), suggesting that these two genes constitute a TA locus. No Pfam domains were identified in Orf2769 and Orf2770, while homologues of these two proteins were annotated as hypothetical proteins found in another Streptomyces sp. by blastp searching against the GenBank non-redundant database (Table S1). Noticeably, the TA pair orf2769/orf2770 does not match any known TA pairs and is only found in Streptomyces sp. The putative TA pair orf2767/orf2766 encodes two small proteins of 65 aa and 89 aa (Figure S5). Orf2766 belongs to the antitoxin Phd family 
(PF02604) and Orf2767 belongs to the toxin ParE family (PF05016) in the Pfam database (Table S1). Orf2767 belongs to the ParE family but shows a very low similarity ( $57 \%$ identity with $6 \%$ coverage) with the previously characterized type-II toxin ParE in conjugative RK2 [32] (Figure S2D). Orf2766 belongs to the Phd family but also shows a low similarity ( $30 \%$ identity with $51 \%$ coverage) with the type-II antitoxin Phd in bacteriophage P1 [33] (Figure S2C). However, overexpressing orf2767 and coexpressing orf 2767 and orf2766 are nontoxic in the E. coli K12 host (Figure 1D and Figure S3C). Thus, two TA pairs, YoeB/YefM and Orf2769/ Orf2770, were chosen for further study.

\begin{tabular}{cccc}
\hline Predicted TA & $\begin{array}{c}\text { Homologous } \\
\text { TA }\end{array}$ & $\begin{array}{c}\text { Toxic test } \\
\text { for toxin }\end{array}$ & $\begin{array}{c}\text { Toxic test } \\
\text { for TA }\end{array}$ \\
\hline Orf5461/ Orf5462 & YoeB / YefM & Yes & No \\
Orf2769/ Orf2770 & None & Yes & No \\
Orf3703/ Orf3704 & VapC / VapB & Yes & No \\
Orf2767/ Orf2766 & ParE/Phd & No & No \\
\hline
\end{tabular}

\section{C}

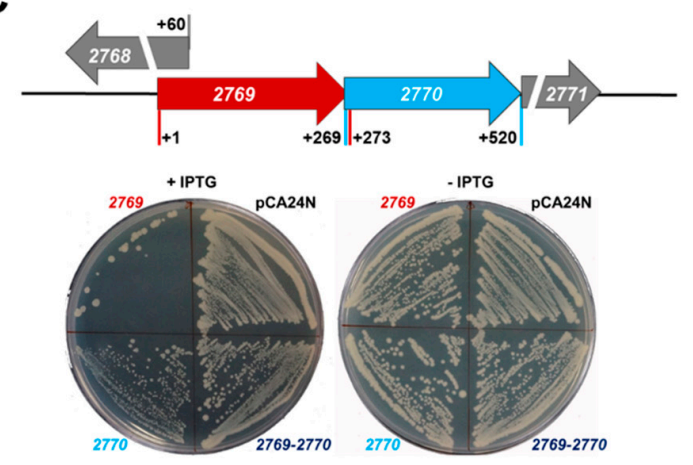

B

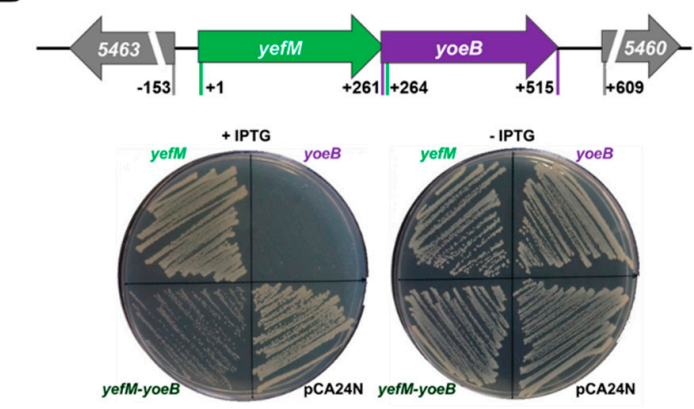

D

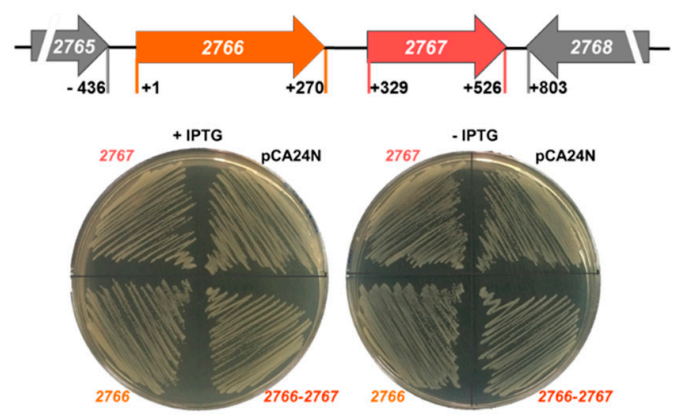

Figure 1. Identification of two TA Pairs. (A) Four potential TA loci in SCSIO 02999 genome were predicted and tested by a toxin test. yoeB-yefM (B), orf2769-orf2770 (C) and orf2767-orf2766 (D) are located in the chromosomal region of SCSIO 02999. The number indicates the relative position to the start codon of yefM, orf 2769 and orf2766 (upper panel). E. coli K-12 BW25113 carrying the pCA24N-based plasmids were streaked on LB plates supplemented with chloramphenicol $(30 \mu \mathrm{g} / \mathrm{mL})$ with or without IPTG $(1 \mathrm{mM})$ (lower panel). Three independent cultures were tested, and only one representative image of each strain is shown here.

\subsection{Characterization of the Type-II TA Pair YoeB/YefM}

To test whether yoeB and yefM are cotranscribed, pHGR01-P-yefM-yoeB containing the $500 \mathrm{bp}$ region upstream of the translational start site of yef $M$ was constructed and transferred into the E. coli K-12 host. Total RNA was used to synthesize cDNA. The results of the RT-PCR analysis indicated the expected sizes of yefM-yoeB, which were consistent with the genomic DNA (gDNA) and cDNA (Figure 2A, lanes 2 and 4). No PCR products were detected in the negative controls (RNA) (Figure 2A, lane 3). These results show that the yoeB and yef $M$ genes are cotranscribed and form a bicistronic operon. In the classical type-II TA systems, the antitoxin protein neutralizes the toxicity of the toxin through a direct protein-protein interaction [34]. To test whether YoeB binds to YefM in vivo, we performed a pull-down assay using pET28b-NHis-yefM-yoeB to coexpress N-terminal hexahistidine-tagged (His-tagged) YefM and an untagged YoeB toxin. Affinity purification using Ni-NTA agarose beads and subsequent Tricine-SDS-PAGE revealed that a small protein was pulled down along with the His-tagged YefM (Figure 2B, lane 1-3), and the small protein was verified by mass spectrometry to be YoeB (Table S2). We also constructed pET28b-yefM-yoeB to coexpress 
untagged YefM and untagged YoeB, and neither protein could bind to Ni-NTA beads (Figure 2B, lane 5-7). These results suggest that YoeB was purified due to its interaction with NHis-YefM. To test whether YoeB is bactericidal or bacteriostatic, we overexpressed yoeB and yoeB $B_{\text {.coli }}$ in the E. coli host, and live/dead staining was performed to check the viability of the cells. As shown in Figure 2C, the majority of cells overexpressing yoeB and yoe $B_{E \text {.coli }}$ remained viable after $2 \mathrm{~h}$ and $4 \mathrm{~h}$ induction with 1 mM IPTG. Moreover, when cells encounter nutritional or environmental stresses, the antitoxin of the type-II TA system is easily degraded by various intracellular proteases, such as Lon, ClpP and ClpX to free the toxins. To test which protease degrades the antitoxin YefM, the pCA24N-NHis-yefM plasmid was transferred into wild-type E. coli. When treated with spectinomycin, N-terminal His-tagged YefM was gradually degraded over time (Figure 2D). Degradation of YefM was also tested in three protease deletion mutant strains, $\Delta l o n, \Delta c l p P$ and $\Delta c l p X$. As shown in Figure 2D and Figure S6, YefM remained stable only in the absence of Lon, suggesting that Lon is mainly responsible for the degradation of YefM. Collectively, these results show that YoeB and YefM constitute a bona fide type-II TA pair.

A

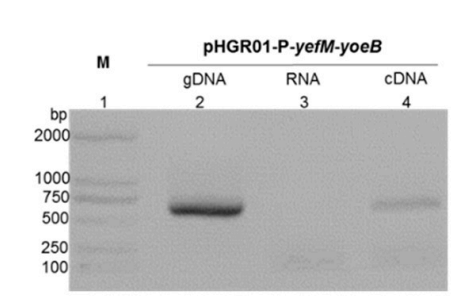

B

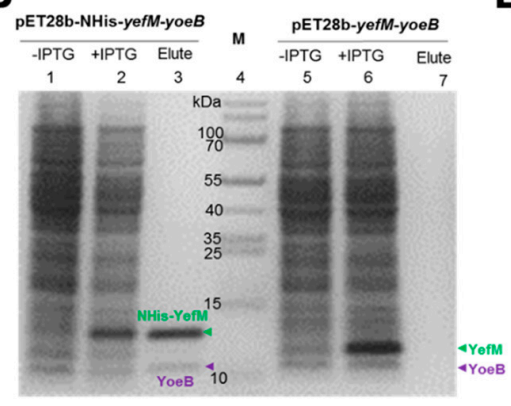

C

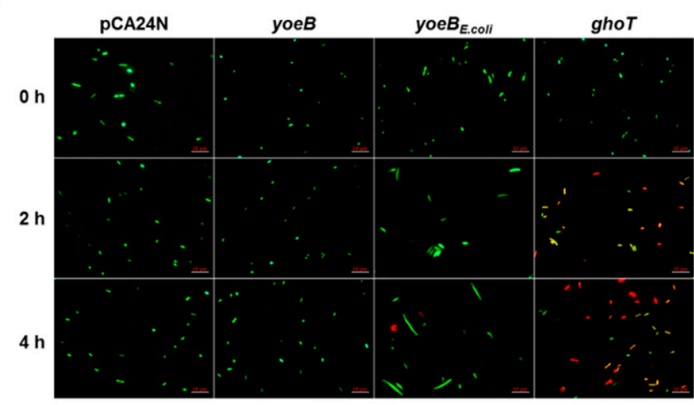

D

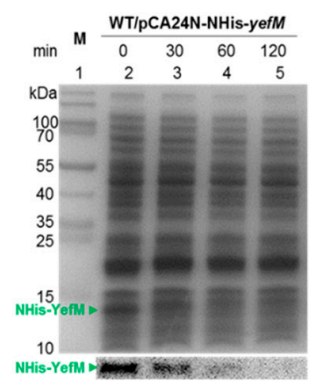

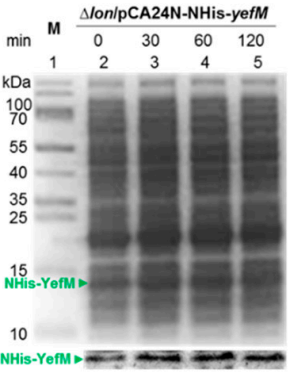

Figure 2. Characterization of the type-II TA pair YoeB/YefM. (A) Total gDNA and RNA were extracted from BW25113/pHGR01-P-yefM-yoeB, and the total RNA was used to synthesize cDNA. PCR was carried out by primer pairs using gDNA (lane 2), RNA (lane 3) and cDNA (lane 4). The DNA marker is in lane 1. (B) His-tagged YefM and untagged YoeB were produced from pET28b-NHis-yefM-yoeB in E. coli BL21. NHis-YefM (10.6 kDa) and YoeB $(9.9 \mathrm{kDa})$ were induced with IPTG $(1 \mathrm{mM})$. YoeB was copurified with NHis-YefM (lane 3). Cells harboring pET28b-yefM-yoeB were used as a negative control. Both untagged YefM and untagged YoeB were induced (lane 6), but neither was bound to the Ni-NTA agarose beads (lane 7). The cells without IPTG induction were used as a negative control (lane 1,5). The protein marker is in lane 4. (C) Cell morphology and live/dead staining of the E. coli K-12 BW25113 overexpressing pCA24N, pCA24N-yoeB, pCA24N-yoeB E.coli and pCA24N-ghoT plasmids at $0 \mathrm{~h}, 2 \mathrm{~h}$ and $4 \mathrm{~h}$. The empty plasmid pCA24N was used as the negative control and pCA24N-ghoT as the positive control. Three independent cultures of each strain were tested, and only representative images are shown here. (D) The E. coli K-12 BW25113 WT and $\Delta l o n$ strains harboring the plasmid pCA24N-NHis-yefM were induced with $0.5 \mathrm{mM}$ IPTG for $30 \mathrm{~min}$, and $1 \%$ spectinomycin $(100 \mu \mathrm{g} / \mathrm{mL})$ was added to the strains to activate a stress response. We collected equivalent quantities of cells at $0 \mathrm{~min}, 30 \mathrm{~min}, 60 \mathrm{~min}$ and $120 \mathrm{~min}$ and then ran Tricine-SDS-PAGE (upper panel) for western blot assays (lower panel). 


\subsection{Cross-Complementation of YoeB/YefM from E. coli and Streptomyces sp.}

Since the YoeB toxin from SCSIO 02999 is still toxic in the E. coli host, we next tested whether the antitoxin YefM is specific for each toxin in different bacterial hosts. Thus, we tested whether there was cross-complementation between the YoeB/YefM TA pairs from E. coli and from SCSIO 02999. We fused the antitoxin yef $M_{E . c o l i}$ upstream of yoeB to construct pCA24N-yoeB-yef $M_{E \text {.coli }}$ and the antitoxin yef $M$ upstream of yoe $B_{E \text {.coli }}$ to construct pCA24N-yoeB $B_{\text {.coli }}-y e f M$. These plasmids were transferred into E. coli $\mathrm{K}-12$ and were induced to overexpress the toxin and antitoxin with $1 \mathrm{mM}$ IPTG. As shown in Figure $3 \mathrm{~A}, \mathrm{~B}$, the antitoxin from E. coli could counteract the toxic effect of YoeB. However, the antitoxin from SCSIO 02999 was unable to counteract the toxic effect of $Y_{\text {YoeB }}$ E.coli. Sequence analysis revealed that the key residues in YoeB and YefM that make contact with each other are different in E. coli and SCSIO 02999 [31] (Figure 3C,D), which may affect the direct contact between the YefM antitoxin from SCSIO 02999 and YoeB from E. coli. Hence, the fact that antitoxin YefM from SCSIO 02999 can only neutralize the YoeB toxin from SCSIO 02999 but not the YoeB toxin from E. coli suggests that the interaction between toxin and antitoxin is optimized in different bacterial hosts.

A

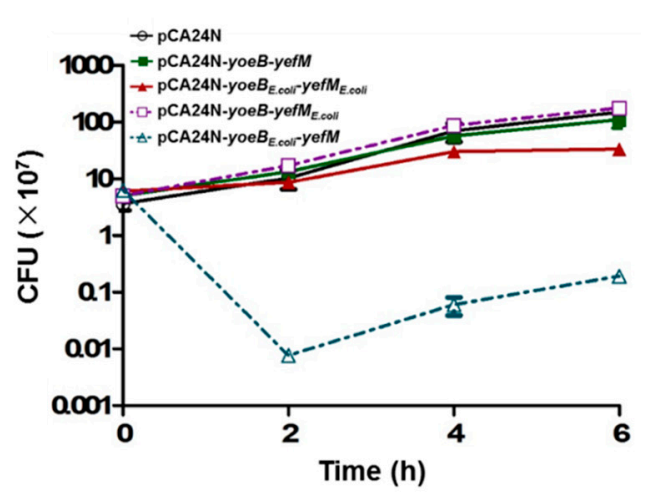

B

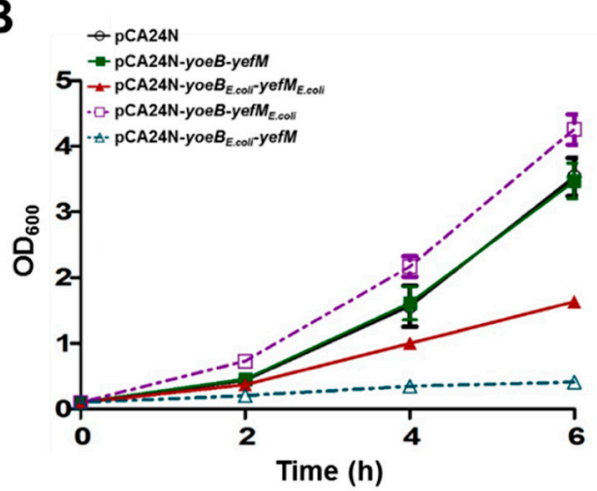

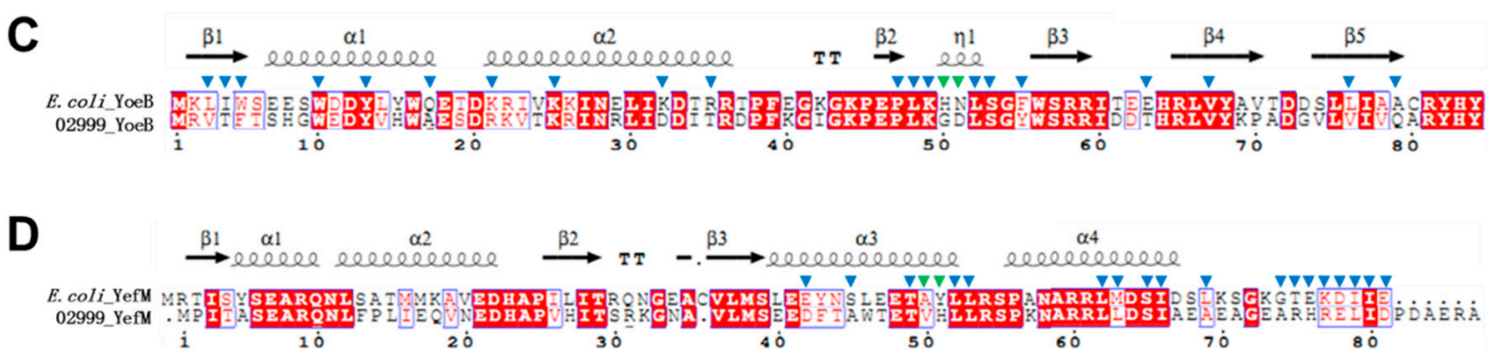

Figure 3. Cross-complementation of YoeB/YefM from E. coli and Streptomyces sp. E. coli K-12 BW25113

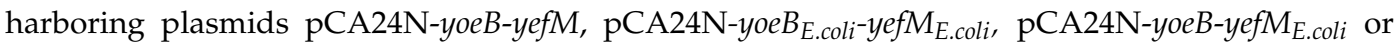

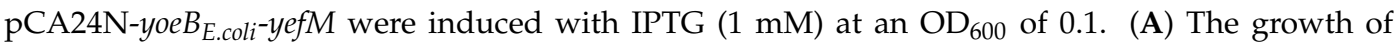
the E. coli strains harboring the pCA24N-based plasmids induced with IPTG $(1 \mathrm{mM})$ at $\mathrm{OD}_{600} \sim 0.1$ was monitored by absorbance at $600 \mathrm{~nm}$. (B) At the times indicated, cell viability (CFUs/mL) was determined on LB agar plates containing chloramphenicol $(30 \mu \mathrm{g} / \mathrm{mL})$. Error bars indicate the standard error of the mean $(n=3)$ in A, B. (C) Comparison of the amino acid sequence of YoeB in E. coli K12 MG1655. (D) Comparison of the amino acid sequence of YefM in E. coli K12 MG1655. YefM has two protomers, and the residues of YoeB participating in direct interactions with different protomers of YefM are marked by blue triangles and green triangles [31].

\subsection{Characterization of a Novel TA Pair Orf2769/Orf2770}

To check whether orf2769 and orf2770 are cotranscribed, pHGR01-P-2769-2770 containing 500 bp of sequence upstream of the translational start site of orf2769 was constructed and transferred into the E. coli K-12 host. Total RNA was used to synthesize cDNA. The RT-PCR analysis showed the expected sizes of orf2769-orf2770, which is consistent with the genomic DNA (gDNA) (Figure 4A, 
lanes 2, 4). No PCR products were detected in the negative controls (RNA) (Figure 4A, lane 3). These results revealed that the orf 2769 and orf 2770 genes are cotranscribed in the E. coli host and form a bicistronic operon. However, a direct interaction between Orf2769 and Orf2770 was not observed using a pull-down assay (Figure 4B) or a bacteria two-hybrid assay (Figure 4C). To test whether Orf2769 is bactericidal or bacteriostatic, we overexpressed orf2769 in the E. coli host, and cell morphology was performed to check the viability of the cells. As shown in Figure 4D, the majority of cells overexpressing orf 2769 became significantly elongated after $8 \mathrm{~h}$ of induction with $1 \mathrm{mM} \mathrm{IPTG}$ and cells overexpressing the Orf2770 also results in slight cell elongation. Cells coexpressing Orf2769 and Orf2770 did not become significantly elongated (Figure 4D). These results are consistent with the above growth inhibition assays. Although no direct interaction between Orf2769 and Orf2770 was detected, Orf2770 is unstable, and Lon protease is mainly responsible for the degradation of Orf2770 (Figure S7). These results suggest that Orf2769 and Orf2770 may constitute a new TA pair.

A

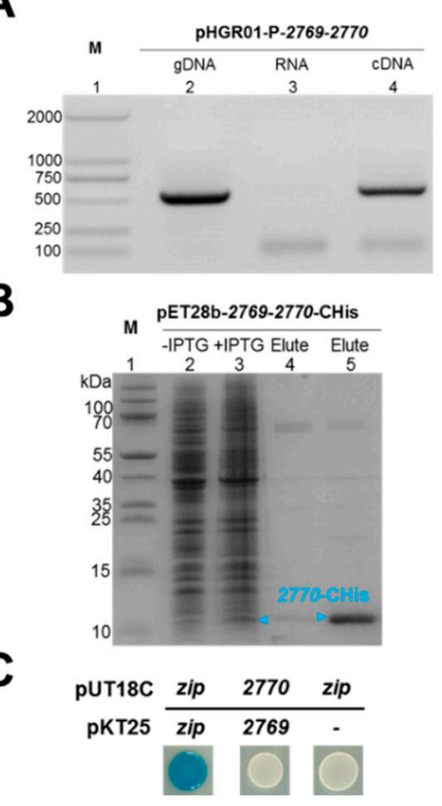

D

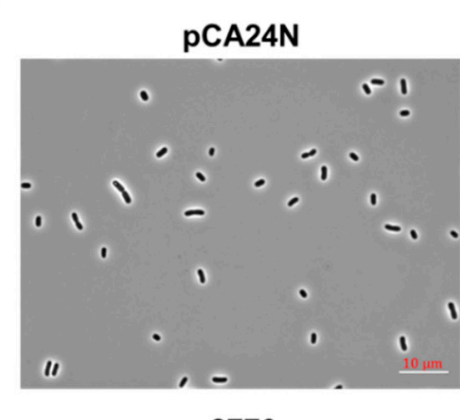

2770

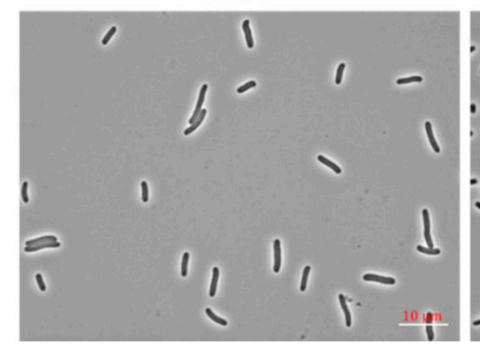

2769

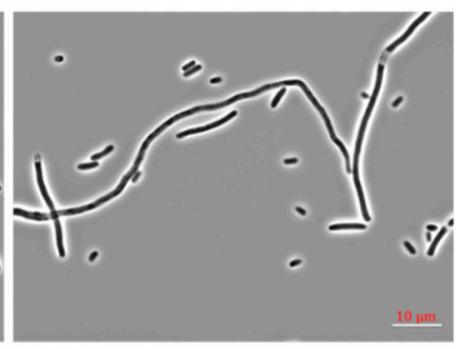

2769-2770

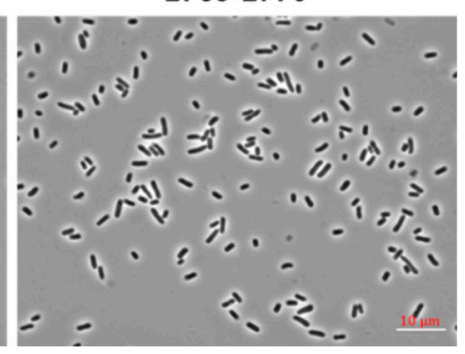

Figure 4. Characterization of a novel TA pair Orf2769/Orf2770. (A) Total gDNA and RNA were extracted from BW25113/pHGR01-P-2769-2770, and the total RNA was used to synthesize cDNA. PCR was carried out by primer pairs using gDNA (lane 2), RNA (lane 3) and cDNA (lane 4). The DNA marker is in lane 1. (B) His-tagged Orf2770 and untagged Orf2769 were produced from pET28b-2769-2770-CHis in an E. coli Rosetta host. Orf2770-CHis was induced (lane 3) and purified, while Orf2769 was unable to be copurified with Orf2770-CHis (lane 4,5). The cells without IPTG induction were used as a negative control (lane 2). The protein marker is in lane 1. (C) Orf2770 was fused to the T18 catalytic domain, and orf 2769 was fused to the T25 fragment to create an in-frame translational fusion of the T25 catalytic domain. Cells harboring pKT25-zip and pUT18C-zip plasmids were used as positive controls, and the cells harboring pKT25 (without an insert) and pUT18C-zip plasmids were used as negative controls. (D) The E. coli K-12 BW25113 harboring pCA24N-2769, pCA24N-2770 and pCA24N-2769-2770 were induced with $1 \mathrm{mM}$ IPTG for $8 \mathrm{~h}$, and then the cell morphology were observed under phase contrast microscope. The empty plasmid pCA24N was used as control. Three independent cultures of each strain were tested, and only representative images are shown here.

\subsection{YoeB/YefM and Orf2769/Orf2770 Both Stabilize Plasmids in E. coli}

Both type-I and -II TA systems have the capability to increase plasmid maintenance, regardless of whether they are chromosome or plasmid origin. To test whether the two TA pairs can stabilize plasmids in E. coli, the high copy number plasmid pCA24N, which requires chloramphenicol to maintain its stability, was used. As expected, E. coli cells harboring pCA24N exhibited a higher 
plasmid loss rate after two days without chloramphenicol. In contrast, approximately $90 \%$ of cells harboring pCA24N-yoeB-yefM or pCA24N-2769-2770 still carried the plasmids after two days (Figure 5). The results indicate that the presence of the TA pairs YoeB/YefM and Orf2769/Orf2770 from deep-sea Streptomyces sp. stabilized plasmid retention in E. coli.

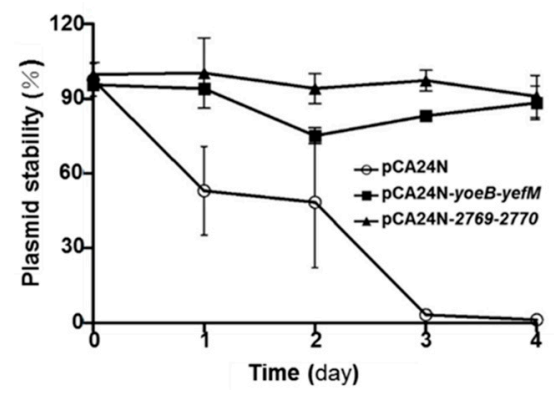

Figure 5. YoeB/YefM and Orf2769/Orf2770 both stabilize plasmids in E. coli. E. coli K-12 BW25113 harboring plasmids pCA24N, pCA24N-yoeB-yefM and pCA24N-2769-2770 were used for the plasmid stability assay. The cultures were diluted 100-fold in LB medium with $0.05 \mathrm{mM}$ IPTG but without any antibiotics and incubated at $37^{\circ} \mathrm{C}$ every $12 \mathrm{~h}$. Then, the cultures were separately plated on LB plates with or without chloramphenicol $(30 \mu \mathrm{g} / \mathrm{mL})$ to determine the number of CFUs every $24 \mathrm{~h}$; this process was repeated for 4 days. The mean and standard deviation from three independent cultures are shown.

\section{Discussion}

In this study, we identified and characterized a type-II TA pair YoeB/YefM and a novel TA pair Orf2769/Orf2770 from SCSIO 02999. These results are as follows: (i) YoeB/YefM and Orf2769/Orf2770 form operons (orf5461/orf5462 and orf2769/2770), and they are cotranscribed; (ii) both YoeB and Orf2769 are toxic; (iii) the cognate antitoxins YefM and Orf2770 can neutralize the toxicity of YoeB and Orf2769, respectively, and the antitoxins are unstable; (iv) YefM counteracts YoeB by direct protein-protein interaction while direct interaction between Orf2769 and Orf2770 is not observed; and (v) both YoeB/YefM and Orf2769/Orf2770 stabilize plasmids in E. coli. TA systems mainly spread between different microorganisms through horizontal gene transfer, and they are suggested to be closely related to the environmental adaptability of microorganisms. Our results show that although YoeB/YefM in SCSIO 02999 shares a high level of amino acid identity with YoeB/YefM in E. coli, the antitoxin YefM from SCSIO 02999 can neutralize the YoeB toxin from SCSIO 02999 but not the toxin from E. coli.; this result is consistent with previous studies, which also suggest that similar TAs have optimized interactions in their hosts, probably in response to different environmental conditions [35,36]. Our previous work demonstrated that the toxin VapC in SCSIO 02999 could cross-activate E. coli TA systems in a partially Lon-dependent manner. However, the qRT-PCR analysis from this study showed that neither YoeB nor 2769 could cross-activate the E. coli TAs (Tables S3 and S4). Collectively, these results suggest that the function and interaction of the toxin and the interaction of TAs from homologous families vary in different hosts.

Streptomyces species produce more than two-thirds of the clinically useful natural antibiotics [37]. Streptomyces sp. is also considered to be a promising bacterial expression system for producing high levels of functional proteins [38,39]. TAs are also common genetic elements in Streptomyces sp.; through bioinformatics analysis, 22 putative TA systems have been identified in Streptomyces coelicolor A3, 27 in Streptomyces avermitilis MA-4680 and 14 in Streptomyces griseus NBRC 13350 [40]. Nevertheless, only a few TA pairs have been identified in Streptomyces sp. by laboratory methods [41,42]. It has been suggested that TAs YoeB/YefM from S. lividans is a powerful tool for producing other proteins of interest in Streptomyces without the use of antibiotics in the production step [43,44]. Thus, TAs are expected to be a valuable tool for the mass production of metabolites in Streptomyces. In this study, the TA pair Orf2769/Orf2770 in SCSIO 02999 was found not to match any known TA pairs and was only found in Streptomyces sp. The biochemical functions of toxins of the TA system include cleaving 
DNA [45,46], mRNA [47], 16S or 23S rRNA and tRNA [48,49], inhibiting gyrase activity [50] and inactivating EF-Tu [51]. Several attempts were made to purify Orf2679 toxin for in vitro functional analysis, but none was successful, mainly due to its high toxicity. Other purification methods, such as releasing antitoxin from the TA complex with denaturation, might be feasible. Nevertheless, functional elucidation of Orf2769 will be helpful to understand the physiological function of the TA pair and to their potential applications.

\section{Experimental Procedures}

\subsection{Bacterial Strains, Plasmids and Growth Conditions}

The bacterial strains, plasmids and all primers used in this study are listed in Table 1 and Table S5. The E. coli strains were cultured at $37^{\circ} \mathrm{C}$ in Luria-Bertani (LB) medium. As previous described, the marine-derived Streptomyces sp. SCSIO 02999 was isolated from a South China Sea sediment sample at a depth of $880 \mathrm{~m}$ [25] and the taxonomy of the strain was analyzed based on the 16S rRNA gene and was deposited in GenBank (accession No. JQ815089) [23]. Chloramphenicol (30 $\mu \mathrm{g} / \mathrm{mL})$ and kanamycin $(50 \mu \mathrm{g} / \mathrm{mL})$ were added when cells harbored plasmids with the indicated resistance gene listed in Table 1 . When required, $\mathrm{mM}$ isopropyl- $\beta$-d-thiogalactoside (IPTG) was added as an expression inducer.

Table 1. Bacterial strains and plasmids used in this study. $\mathrm{Cm}^{\mathrm{R}}, \mathrm{Km}^{\mathrm{R}}$ and $A m p^{\mathrm{R}}$ indicate chloramphenicol, kanamycin and ampicillin resistance respectively. $\mathrm{P}$ indicates promoter.

\begin{tabular}{|c|c|c|}
\hline Bacterial Strains/Plasmids & Genotype or Description & Source \\
\hline \multicolumn{3}{|l|}{ Streptomyces sp. SCSIO 02999} \\
\hline wild-type & A marine-derived Streptomyces sp., cultured in AM6 medium & [29] \\
\hline \multicolumn{3}{|l|}{ E. coli strains } \\
\hline K-12 BW25113 & lacI $^{\mathrm{q}} r r n B_{\mathrm{T} 14} \Delta l a c Z_{\mathrm{WJ} 16} h s d R 514 \Delta a r a B A D_{\mathrm{AH} 33} \Delta r h a B A D_{\mathrm{LD} 78} r p h-1$ & [52] \\
\hline K-12 BW25113 $\Delta l o n$ & $\Delta$ lon $\Delta \mathrm{km}^{R}$ & {$[52]$} \\
\hline $\mathrm{K}-12 \mathrm{BW} 25113 \Delta c l p P$ & $\Delta c l p P \Delta k m^{R}$ & [52] \\
\hline $\mathrm{K}-12 \mathrm{BW} 25113 \Delta c l p X$ & $\Delta c l p X \Delta k m^{R}$ & [52] \\
\hline BL21(DE3) & $\mathrm{F}^{-}$omp $T h s d S_{B}\left(r_{B}{ }^{-} m_{B}{ }^{-}\right)$gal $d c m \lambda(\mathrm{DE} 3) \Omega \mathrm{P}_{\text {tacUV5 }}:: T 7$ polymerase & Novagen \\
\hline WM3064 & $\begin{array}{l}\text { thrB1004 pro thi rpsL hsdS lacZ } \Delta \text { M15 RP4-1360) } \Delta(\text { araBAD)567 } \Delta \text { dapA1341::[erm } \\
\operatorname{pir}(\mathrm{wt})]\end{array}$ & $\begin{array}{l}\text { W. Metcalf, } \\
\text { UIUC }\end{array}$ \\
\hline Rosetta (DE3) & $\begin{array}{l}\mathrm{F}^{-} \operatorname{omp} \mathrm{T} h d \mathrm{~S}_{\mathrm{B}}\left(\mathrm{r}_{\mathrm{B}}^{-} \mathrm{m}_{\mathrm{B}}^{-}\right) \text {gal dcm (DE3) } p R A R E(\arg U, \operatorname{argW}, \text { ilex, glyT, leuW, } \\
\operatorname{proL})\left(\mathrm{Cm}^{\mathrm{R}}\right)\end{array}$ & Novagen \\
\hline BTH101 & $\mathrm{F}^{-}$, cya-99,araD139,galE15,galK16,rpsL1(Str $\left.{ }^{\mathrm{R}}\right), h s d R 2$, mcrA1, mcrB1 & {$[53]$} \\
\hline \multicolumn{3}{|l|}{ Plasmids } \\
\hline pCA24N & $\mathrm{Cm}^{\mathrm{R}}$; lacI ${ }^{\mathrm{q}}$, IPTG inducible expression vector & [54] \\
\hline pCA24N-2769 & $\begin{array}{l}\text { q q } \\
\text { Cm }^{R} \text {; lacI }{ }^{\mathrm{q}}, \mathrm{P}_{\mathrm{T} 5-\text { lac: }: 2769} \\
\text { q, PT5-lac::vapB q, PT5-lac::vapB }\end{array}$ & this study \\
\hline pCA24N-2770 & $\mathrm{Cm}^{\mathrm{R}} ; \mathrm{lacI}^{\mathrm{q}}, \mathrm{P}_{\mathrm{T} 5-\mathrm{lac}}:: 2770$ & this study \\
\hline pCA24N-2769-2770 & $\mathrm{Cm}^{\mathrm{R}} ;$ lacI ${ }^{\mathrm{q}}, \mathrm{P}_{\mathrm{T} 5-\mathrm{lac}}:: 2769-2770$ & this study \\
\hline pCA24N-yoeB & $\mathrm{Cm}^{\mathrm{R}} ; \mathrm{lacI}^{\mathrm{q}}, \mathrm{P}_{\mathrm{T} 5-\mathrm{lac}}::$ yoeB & this study \\
\hline pCA24N-yefM & $\mathrm{Cm}^{\mathrm{R}} ;$ lacI $^{\mathrm{q}}, \mathrm{P}_{\mathrm{T} 5 \text {-lac }}::$ yefM & this study \\
\hline pCA24N-yoeB-yefM & $\mathrm{Cm}^{\mathrm{R}}$; lacI ${ }^{\mathrm{q}}, \mathrm{P}_{\mathrm{T} 5-\mathrm{lac}}::$ yoeB-yefM & this study \\
\hline pCA24N-yoeB E.coli-yefM $M_{E . c o l i}$ & $\mathrm{Cm}^{\mathrm{R}} ;$ lacI $^{\mathrm{q}}, \mathrm{P}_{\mathrm{T} 5 \text {-lac }}:$ yoe $B_{\text {E.coli-yefM }}$ E.coli & this study \\
\hline pCA24N-yoeB-yefM $M_{\text {E.coli }}$ & $\mathrm{Cm}^{\mathrm{R}} ;$ lacI ${ }^{\mathrm{q}}, \mathrm{P}_{\mathrm{T} 5-\mathrm{lac}}::$ yoeB-yefM $\mathrm{E}_{\text {E.coli }}$ & this study \\
\hline pCA24N-yoeB $B_{\text {E.coli-yefM }}$ & $\mathrm{Cm}^{\mathrm{R}}$; lacI ${ }^{\mathrm{q}}, \mathrm{P}_{\mathrm{T} 5-\mathrm{lac}}::$ yoe $B_{\text {E.coli }}-$ yefM & this study \\
\hline pCA24N-yoeB ${ }_{\text {E.coli }}$ & $\mathrm{Cm}^{\mathrm{R}}$; lacI ${ }^{\mathrm{q}}, \mathrm{P}_{\mathrm{T} 5 \text {-lac }}: \because$ yoe $B_{\text {E.coli }}$ & {$[54]$} \\
\hline pCA24N-ghoT & $\mathrm{Cm}^{\mathrm{R}}$; lacI ${ }^{\mathrm{q}}, \mathrm{P}_{\mathrm{T} 5-\mathrm{lac}}::$ ghoT & [54] \\
\hline pHGR01 & $\mathrm{Km}^{\mathrm{R}}$; R6K ori, promoterless lacZ reporter vector & [55] \\
\hline pHGR01-P-2769-2770 & $\mathrm{Km}^{\mathrm{R}}$; R6K ori, fused 2769-2770 promoter in pHGR01 & this study \\
\hline pHGR01-P-yoeB-yefM & $\mathrm{Km}^{\mathrm{R}} ; \mathrm{R} 6 \mathrm{~K}$ ori, fused yoeB-yefM promoter in pHGR01 & this study \\
\hline pET28b & $\mathrm{Km}^{\mathrm{R}}$; lacI $\mathrm{q}^{\mathrm{q}}$ IPTG inducible expression vector & Novagen \\
\hline pET28b-NHis-yefM-yoeB & $\mathrm{Km}^{\mathrm{R}}$; lacI $\mathrm{q}, \mathrm{pET} 28 \mathrm{~b} \mathrm{P}_{T 7-l a c}::$ yoeB-yefM with $\mathrm{N}$-terminal His-tagged & this study \\
\hline pET28b-yefM-yoeB & $\mathrm{Km}^{\mathrm{R}}$; lacI ${ }^{\mathrm{q}}, \mathrm{pET} 28 \mathrm{~b} \mathrm{P}_{\mathrm{T} \text {-lac }}::$ yoeB-yefM without His-tagged & this study \\
\hline pET28b-2769-2770-CHis & $\mathrm{Km}^{\mathrm{R}}$; lacI ${ }^{\mathrm{q}}, \mathrm{pET} 28 \mathrm{~b} \mathrm{P}_{\text {T7-lac }}:: 2769-2770$ with C-terminal His-tagged & this study \\
\hline pKT25 & $\mathrm{Km}^{\mathrm{R}}$; encoding $\mathrm{T} 25$ fragment & [56] \\
\hline pKT25-2769 & $\mathrm{Km}^{\mathrm{R}} ; 2769$ was fused to the $\mathrm{C}$ termini of adenylate cyclase in pKT25 & this study \\
\hline pUT18C & $\mathrm{Amp}_{\mathrm{D}}^{\mathrm{R}}$; encoding T18 fragment & [56] \\
\hline pUT18C-2770 & $A m p^{R} ; 2770$ was fused to the $C$ termini of adenylate cyclase in pUT18C & this study \\
\hline
\end{tabular}




\subsection{Construction of Expression Plasmids}

The open reading frames of yoeB, yefM, yoeB-yefM, orf2769, orf2770, orf2769-orf2770, yoeB-yefM $M_{\text {E.coli }}$ and yoeB $B_{E . c o l i}-y e f M$ were amplified with primer pairs shown in Supplementary Table S5. Then, the purified fragments were digested with StuI restriction enzymes and ligated into the pCA24N expression plasmid by ClonExpress ${ }^{\mathrm{TM}}$ II One Step Cloning Kit (Vazyme Biotech, Piscataway, NJ, USA), to generate pCA24N-yoeB, pCA24N-yefM, pCA24N-yoeB-yefM, pCA24N-2769, pCA24N-2770,

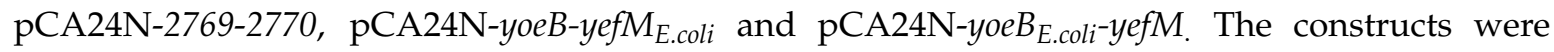
confirmed by PCR followed by DNA sequencing using primers pCA24N-f/r. The pET28b, pHGR01, pKT25 and pUT18C recombinant plasmids were constructed following similar steps. Detailed information on the primer pairs used for PCR amplification, restriction enzyme sites used in the digestion of the PCR products, and the primers used for PCR sequencing, is listed in Supplementary Table S5.

\subsection{Protein Expression and Purification}

YefM plasmids were constructed with or without hexahistidine tagged the $N$-terminus and YoeB was inserted into plasmid pET28b; the constructed plasmids pET28b-NHis-yefM-yoeB and pET28b-yefM-yoeB were then transformed into E. coli BL21. Overnight cultures of the strains were diluted with LB containing kanamycin $(50 \mu \mathrm{g} / \mathrm{mL})$ at an $\mathrm{OD}_{600}$ of 0.1 , and protein expression was induced in the strains with the addition of IPTG $(1 \mathrm{mM})$ when cultures reached an $\mathrm{OD}_{600}$ of 1.0 . After inducing with IPTG for $4 \mathrm{~h}$, cells were collected and resuspended in lysis buffer (50 mM potassium phosphate buffer ( $\mathrm{pH} 8.0$ ), $300 \mathrm{mM} \mathrm{NaCl}$, and a protease inhibitor cocktail (Sigma-Aldrich, St. Louis, MO, USA)). The cells were lysed using a constant systems cell disruptor (Constant Systems Limited, Northants, UK) twice with $30 \mathrm{MPa}$. Then, the lysed cells were centrifuged at high speed ( 9000 rpm) for $1 \mathrm{~h}$, and the supernatant was incubated with Ni-NTA resin (Qiagen, Valencia, CA, USA) according to the manufacturer's protocol. The protein concentration was measured by the Bi Yuntian BCA assay kit (Bioteke Corporation, Haimen, Jiangsu, China). A total of $20 \mu \mathrm{g}$ of protein from each sample was loaded for Tricine-SDS-PAGE.

\subsection{RNA Isolation, RT-PCR and $q R T-P C R$}

Total RNA was isolated following the protocol of the QIAGEN RNeasy Mini Kit (Tiangen, Beijing, China). A total of $1 \mu \mathrm{g}$ of total RNA was used to conduct reverse transcription reactions to synthesize cDNA (Promega, Madison, WI, USA). For the cotranscription assay, the gene-specific primers shown in Table S5 were used for PCR amplification. Then, $50 \mathrm{ng}$ of cDNA was used to conduct qRT-PCR with the SYBR green reaction mix (Applied Biosystems Step One ${ }^{\mathrm{TM}}$ Real-Time PCR System, Invitrogen, Carlsbad, CA, USA). All of the primers for qRT-PCR are listed in Table S5. The level of the rrsG transcript was used as a reference to normalize the gene expression data. Lower Ct (cycle threshold) values indicate higher expression levels. Fold changes in the transcripts of various targets with pCA24N, pCA24N-2769 or pCA24N-yoeB were calculated as follows: $\log _{2}$ ((Ct target(pCA24N-2769)-Ct rrsG(pCA24N-2769))-((Ct target(pCA24N)-Ct rrsG(pCA24N)) or $\log _{2}((\mathrm{Ct} \operatorname{target}(\mathrm{pCA} 24 \mathrm{~N}-$ yoeB $)-\mathrm{Ct}$ rrsG(pCA24N-yoeB))-((Ct target(pCA24N)-Ct rrsG(pCA24N)).

\subsection{Live/Dead Staining}

Overnight cultures of E. coli K-12 BW25113 carrying the empty pCA24N, pCA24N-yoeB, pCA24N-yoeB $B_{\text {.coli }}$ and pCA24N-ghoT plasmids were diluted with LB with chloramphenicol $(30 \mu \mathrm{g} / \mathrm{mL})$ at an $\mathrm{OD}_{600}$ of 0.1 , and IPTG $(1 \mathrm{mM})$ was added at $\mathrm{OD}_{600} \sim 0.5$ to induce expression of proteins. Cells were collected at $0 \mathrm{~h}, 2 \mathrm{~h}$ and $4 \mathrm{~h}$ by centrifugation at $3000 \times \mathrm{g}$ for $5 \mathrm{~min}$, and the cells were resuspended in $300 \mu \mathrm{L}$ of phosphate buffered saline (PBS, pH 7.4). Cells were stained with the LIVE/DEAD ${ }^{\mathrm{TM}}$ BacLight ${ }^{\mathrm{TM}}$ Bacterial Viability Kit, according to the manufacturer's instructions. Cells were stained with $1 \mu \mathrm{L}$ SYTO 9 nucleic acid stain $(300 \mu \mathrm{L}, 3.34 \mathrm{mM}$ in DMSO $)$ and $1 \mu \mathrm{L}$ propidium iodide $(300 \mu \mathrm{L}$, 
$20 \mathrm{mM}$ in DMSO) and incubated in the dark for $10 \mathrm{~min}$. Then, the stained cells were collected and rinsed once with PBS before resuspending in $50 \mu \mathrm{L}$ of PBS.

\subsection{Western Blot Analysis}

The pCA24N plasmid was constructed with NHis-yefM or NHis-2770 and transformed into E. coli K-12 BW25113 WT, $\Delta l o n, \Delta c l p P$ and $\Delta c l p X$ strains. The strains were incubated overnight in LB to reach exponential growth $\left(\mathrm{OD}_{600} \sim 1.0\right)$, then the cells were induced with $1 \% 0.5 \mathrm{mM}$ IPTG for $30 \mathrm{~min}$ at $37^{\circ} \mathrm{C}$. All cells were collected by low-speed centrifugation and resuspended in LB medium until the $\mathrm{OD}_{600}$ reached 1.0. The stress response was activated with $1 \%$ spectinomycin $(100 \mu \mathrm{g} / \mathrm{mL})$ and equal numbers of gathered cells at $0 \mathrm{~min}, 30 \mathrm{~min}, 60 \mathrm{~min}$ and $120 \mathrm{~min}$. Protein samples were separated by Trisine-SDS-PAGE and transferred to a PVDF membrane. Western blot analysis was performed with a primary antibody raised against the His-tag and a horseradish-peroxidase-conjugated goat anti-mouse secondary antibody.

\subsection{BACTH Assay}

For BACTH assays, the coding region of orf 2770 was cloned into pUT18C, and orf 2769 was cloned into pKT25. The constructed plasmids were transformed into E. coli BTH101 (cya-99) competent cells. Cotransformed cells were plated on LB agar plates supplemented with kanamycin $(50 \mu \mathrm{g} / \mathrm{mL})$, ampicillin $(100 \mu \mathrm{g} / \mathrm{mL})$ and X-gal $(20 \mu \mathrm{g} / \mathrm{mL})$. The cells were cultivated at $30{ }^{\circ} \mathrm{C}$ for $24 \mathrm{~h}$. The cells harboring pKT25 (without an insert) and pUT18C-zip (fused with a leucine zipper protein) plasmids were used as negative controls, and the cells harboring pKT25 (without an insert) and the cells harboring pKT25-zip (fused with a leucine zipper protein) and pUT18C-zip plasmids were used as positive controls [55].

\subsection{Plasmid Stability Test}

The E. coli K-12 BW25113 strains harboring plasmids pCA24N, pCA24N-yoeB-yefM and pCA24N-2769-2770 were incubated on LB plates with chloramphenicol $(30 \mu \mathrm{g} / \mathrm{mL})$ at $37^{\circ} \mathrm{C}$. Overnight cultures $(1 \mathrm{~mL})$ were washed twice with $\mathrm{LB}$, resuspended in $1 \mathrm{~mL}$ of fresh $\mathrm{LB}$ and $1 \%$ of the culture reincubated with $2 \mathrm{~mL}$ of $\mathrm{LB}$ in sterile tubes. The initial CFU number of the strains was calculated and plated on LB plates and LB + chloramphenicol $(30 \mu \mathrm{g} / \mathrm{mL})$ plates separately. The strains were cultured at $37^{\circ} \mathrm{C}$. One percent of the culture was subcultured in sterile tubes with $0.05 \mathrm{mM} \mathrm{IPTG}$ every $12 \mathrm{~h}$, and the CFU of the strains were calculated on LB plates and LB with chloramphenicol $(30 \mu \mathrm{g} / \mathrm{mL})$ plates every $24 \mathrm{~h}$. The process was repeated for four days.

\subsection{Sequence Alignment}

Sequence analysis of the three TA pairs was performed by pfam database (http:/ / pfam.xfam.org/ search\#tabview=tab0) and GenBank non-redundant database (https:/ /blast.ncbi.nlm.nih.gov/Blast. cgi).

Supplementary Materials: The Supplementary Materials are available online at http:/ / www.mdpi.com/1660$3397 / 17 / 4 / 211 /$ s1.

Author Contributions: Conceptualization, Y.G. and X.W.; Data curation, W.Z., J.Y. and K.T.; Formal analysis, W.Z., Y.G. and X.W.; Methodology, W.Z., J.Y. and Y.L.; Visualization, W.Z.; Writing—original draft, W.Z., Y.G. and X.W.; Writing—review \& editing, Y.G. and X.W.

Funding: This work was supported by the National Key R\&D Program of China (2018YFC1406500 and 2017YFC0506303), by the National Science Foundation of China (31625001 and 31500025), by National Science Foundation of Guangdong Province (2017A030313125).

Acknowledgments: We are grateful for Changsheng Zhang from our institute for his generosity in providing us the Streptomyces sp. SCSIO 02999 and its genomic sequence. We also thank Shikun Dai (the Equipment Public Service Center, SCSIO.CAS) for assistance in the MALDI-TOF mass spectrometric analyses. XW is a 1000-Youth Elite Program recipient in China. 
Conflicts of Interest: The authors declare no conflict of interest.

\section{References}

1. Harms, A.; Brodersen, D.E.; Mitarai, N.; Gerdes, K. Toxins, targets, and triggers: An overview of toxin-antitoxin biology. Mol. Cell 2018, 70, 768-784. [CrossRef] [PubMed]

2. Hayes, F.; Van Melderen, L. Toxins-antitoxins: Diversity, evolution and function. Crit. Rev. Biochem. Mol. 2011, 46, 386-408. [CrossRef] [PubMed]

3. Hall, A.M.; Gollan, B.; Helaine, S. Toxin-antitoxin systems: Reversible toxicity. Curr. Opin. Microbiol. 2017, 36, 102-110. [CrossRef] [PubMed]

4. Page, R.; Peti, W. Toxin-antitoxin systems in bacterial growth arrest and persistence. Nat. Chem. Biol. 2016, 12, 208-214. [CrossRef]

5. Chan, W.T.; Moreno-Cordoba, I.; Yeo, C.C.; Espinosa, M. Toxin-antitoxin genes of the Gram-positive pathogen Streptococcus pneumoniae: So few and yet so many. Microbiol. Mol. Biol. Rev. MMBR 2012, 76, 773-791. [CrossRef]

6. Zaychikova, M.V.; Zakharevich, N.V.; Sagaidak, M.O.; Bogolubova, N.A.; Smirnova, T.G.; Andreevskaya, S.N.; Larionova, E.E.; Alekseeva, M.G.; Chernousova, L.N.; Danilenko, V.N. Mycobacterium tuberculosis Type II toxin-antitoxin systems: Genetic polymorphisms and functional properties and the possibility of their use for genotyping. PLoS ONE 2015, 10, e0143682. [CrossRef]

7. Lobato-Marquez, D.; Moreno-Cordoba, I.; Figueroa, V.; Diaz-Orejas, R.; Garcia-del Portillo, F. Distinct type I and type II toxin-antitoxin modules control Salmonella lifestyle inside eukaryotic cells. Sci. Rep. 2015, 5, 9374. [CrossRef] [PubMed]

8. Wood, T.L.; Wood, T.K. The HigB/HigA toxin/antitoxin system of Pseudomonas aeruginosa influences the virulence factors pyochelin, pyocyanin, and biofilm formation. Microbiol. Open 2016, 5, 499-511. [CrossRef]

9. Li, G.; Shen, M.; Lu, S.; Le, S.; Tan, Y.; Wang, J.; Zhao, X.; Shen, W.; Guo, K.; Yang, Y.; et al. Identification and characterization of the HicAB toxin-antitoxin system in the opportunistic pathogen Pseudomonas aeruginosa. Toxins 2016, 8, 113. [CrossRef]

10. Muthuramalingam, M.; White, J.C.; Murphy, T.; Ames, J.R.; Bourne, C.R. The toxin from a ParDE toxin-antitoxin system found in Pseudomonas aeruginosa offers protection to cells challenged with anti-gyrase antibiotics. Mol. Microbiol. 2019, 111, 441-454. [CrossRef] [PubMed]

11. Fineran, P.C.; Blower, T.R.; Foulds, I.J.; Humphreys, D.P.; Lilley, K.S.; Salmond, G.P. The phage abortive infection system, ToxIN, functions as a protein-RNA toxin-antitoxin pair. Proc. Natl. Acad. Sci. USA 2009, 106, 894-899. [CrossRef] [PubMed]

12. Rao, F.; Short, F.L.; Voss, J.E.; Blower, T.R.; Orme, A.L.; Whittaker, T.E.; Luisi, B.F.; Salmond, G.P. Co-evolution of quaternary organization and novel RNA tertiary interactions revealed in the crystal structure of a bacterial protein-RNA toxin-antitoxin system. Nucleic Acids Res. 2015, 43, 9529-9540. [CrossRef]

13. Aakre, C.D.; Phung, T.N.; Huang, D.; Laub, M.T. A bacterial toxin inhibits DNA replication elongation through a direct interaction with the beta sliding clamp. Mol. Cell 2013, 52, 617-628. [CrossRef] [PubMed]

14. Piscotta, F.J.; Jeffrey, P.D.; Link, A.J. ParST is a widespread toxin-antitoxin module that targets nucleotide metabolism. Proc. Natl. Acad. Sci. USA 2019, 116, 826-834. [CrossRef] [PubMed]

15. Jankevicius, G.; Ariza, A.; Ahel, M.; Ahel, I. The toxin-antitoxin system DarTG catalyzes reversible ADP-ribosylation of DNA. Mol. Cell 2016, 64, 1109-1116. [CrossRef] [PubMed]

16. Jurenas, D.; Chatterjee, S.; Konijnenberg, A.; Sobott, F.; Droogmans, L.; Garcia-Pino, A.; Van Melderen, L. AtaT blocks translation initiation by $\mathrm{N}$-acetylation of the initiator tRNA(fMet). Nat. Chem. Biol. 2017, 13, 640-646. [CrossRef] [PubMed]

17. Jurenas, D.; Van Melderen, L.; Garcia-Pino, A. Mechanism of regulation and neutralization of the AtaR-AtaT toxin-antitoxin system. Nat. Chem. Biol. 2019, 15, 285-294. [CrossRef] [PubMed]

18. Marsan, D.; Place, A.; Fucich, D.; Chen, F. Toxin-antitoxin systems in estuarine Synechococcus strain CB0101 and their transcriptomic responses to environmental stressors. Front. Microbiol. 2017, 8, 1213. [CrossRef] [PubMed]

19. Wen, Y.; Behiels, E.; Felix, J.; Elegheert, J.; Vergauwen, B.; Devreese, B.; Savvides, S.N. The bacterial antitoxin HipB establishes a ternary complex with operator DNA and phosphorylated toxin HipA to regulate bacterial persistence. Nucleic Acids Res. 2014, 42, 10134-10147. [CrossRef] [PubMed] 
20. Yao, J.; Guo, Y.; Zeng, Z.; Liu, X.; Shi, F.; Wang, X. Identification and characterization of a HEPN-MNT family type II toxin-antitoxin in Shewanella oneidensis. Microb. Biotechnol. 2015, 8, 961-973. [CrossRef] [PubMed]

21. Yao, J.; Guo, Y.; Wang, P.; Zeng, Z.; Li, B.; Tang, K.; Liu, X.; Wang, X. Type II toxin/antitoxin system ParESO /CopASO stabilizes prophage CP4So in Shewanella oneidensis. Environ. Microbiol. 2018, 20, 1224-1239. [CrossRef] [PubMed]

22. Schippers, A.; Neretin, L.N.; Kallmeyer, J.; Ferdelman, T.G.; Cragg, B.A.; Parkes, R.J.; Jorgensen, B.B. Prokaryotic cells of the deep sub-seafloor biosphere identified as living bacteria. Nature 2005, 433, 861-864. [CrossRef] [PubMed]

23. Guo, Y.; Yao, J.; Sun, C.; Wen, Z.; Wang, X. Characterization of the deep-sea Streptomyces sp. SCSIO 02999 derived VapC/VapB toxin-antitoxin system in Escherichia coli. Toxins 2016, 8, 195. [CrossRef] [PubMed]

24. Lee, C.T.; Chen, I.T.; Yang, Y.T.; Ko, T.P.; Huang, Y.T.; Huang, J.Y.; Huang, M.F.; Lin, S.J.; Chen, C.Y.; Lin, S.S.; et al. The opportunistic marine pathogen Vibrio parahaemolyticus becomes virulent by acquiring a plasmid that expresses a deadly toxin. Proc. Natl. Acad. Sci. USA 2015, 112, 10798-10803. [CrossRef] [PubMed]

25. Zhang, Q.B.; Mandi, A.; Li, S.M.; Chen, Y.C.; Zhang, W.J.; Tian, X.P.; Zhang, H.B.; Li, H.X.; Zhang, W.M.; Zhang, S.; et al. N-N-coupled indolo-sesquiterpene atropo-diastereomers from a marine-derived actinomycete. Eur. J. Org. Chem. 2012, 2012, 5256-5262. [CrossRef]

26. Saha, S.; Zhang, W.; Zhang, G.; Zhu, Y.; Chen, Y.; Liu, W.; Yuan, C.; Zhang, Q.; Zhang, H.; Zhang, L.; et al. Activation and characterization of a cryptic gene cluster reveals a cyclization cascade for polycyclic tetramate macrolactams. Chem. Sci. 2017, 8, 1607-1612. [CrossRef]

27. Zhang, Q.; Li, H.; Yu, L.; Sun, Y.; Zhu, Y.; Zhu, H.; Zhang, L.; Li, S.M.; Shen, Y.; Tian, C.; et al. Characterization of the flavoenzyme $\mathrm{XiaK}$ as an N-hydroxylase and implications in indolosesquiterpene diversification. Chem. Sci. 2017, 8, 5067-5077. [CrossRef]

28. Chen, R.; Zhang, Q.; Tan, B.; Zheng, L.; Li, H.; Zhu, Y.; Zhang, C. Genome mining and activation of a silent PKS/NRPS gene cluster direct the production of totopotensamides. Org. Lett. 2017, 19, 5697-5700. [CrossRef]

29. Li, H.; Zhang, Q.; Li, S.; Zhu, Y.; Zhang, G.; Zhang, H.; Tian, X.; Zhang, S.; Ju, J.; Zhang, C. Identification and characterization of xiamycin A and oxiamycin gene cluster reveals an oxidative cyclization strategy tailoring indolosesquiterpene biosynthesis. J. Am. Chem. Soc. 2012, 134, 8996-9005. [CrossRef]

30. Sevin, E.W.; Barloy-Hubler, F. RASTA-Bacteria: A web-based tool for identifying toxin-antitoxin loci in prokaryotes. Genome Biol. 2007, 8, R155. [CrossRef]

31. Kamada, K.; Hanaoka, F. Conformational change in the catalytic site of the ribonuclease YoeB toxin by YefM antitoxin. Mol. Cell 2005, 19, 497-509. [CrossRef] [PubMed]

32. Roberts, R.C.; Helinski, D.R. Definition of a minimal plasmid stabilization system from the broad-host-range plasmid RK2. J. Bacteriol. 1992, 174, 8119-8132. [CrossRef]

33. Gazit, E.; Sauer, R.T. Stability and DNA binding of the phd protein of the phage P1 plasmid addiction system. J. Biol. Chem. 1999, 274, 2652-2657. [CrossRef] [PubMed]

34. Brown, B.L.; Wood, T.K.; Peti, W.; Page, R. Structure of the Escherichia coli antitoxin MqsA (YgiT/b3021) bound to its gene promoter reveals extensive domain rearrangements and the specificity of transcriptional regulation. J. Biol. Chem. 2011, 286, 2285-2296. [CrossRef] [PubMed]

35. Nieto, C.; Cherny, I.; Khoo, S.K.; de Lacoba, M.G.; Chan, W.T.; Yeo, C.C.; Gazit, E.; Espinosa, M. The yefM-yoeB toxin-antitoxin systems of Escherichia coli and Streptococcus pneumoniae: Functional and structural correlation. J. Bacteriol. 2007, 189, 1266-1278. [CrossRef] [PubMed]

36. Zheng, C.; Xu, J.; Ren, S.; Li, J.; Xia, M.; Chen, H.; Bei, W. Identification and characterization of the chromosomal yefM-yoeB toxin-antitoxin system of Streptococcus suis. Sci. Rep. 2015, 5, 13125. [CrossRef]

37. Bibb, M.J. Understanding and manipulating antibiotic production in actinomycetes. Biochem. Soc. Trans. 2013, 41, 1355-1364. [CrossRef]

38. Vrancken, K.; Anne, J. Secretory production of recombinant proteins by Streptomyces. Future Microbiol. 2009, 4, 181-188. [CrossRef]

39. Anne, J.; Maldonado, B.; Van Impe, J.; Van Mellaert, L.; Bernaerts, K. Recombinant protein production and streptomycetes. J. Biotechnol. 2012, 158, 159-167. [CrossRef]

40. Jia, N.; Ding, M.Z.; Luo, H.; Gao, F.; Yuan, Y.J. Complete genome sequencing and antibiotics biosynthesis pathways analysis of Streptomyces lydicus 103. Sci. Rep. 2017, 7, 44786. [CrossRef] 
41. Sevillano, L.; Diaz, M.; Yamaguchi, Y.; Inouye, M.; Santamaria, R.I. Identification of the first functional toxin-antitoxin system in Streptomyces. PLoS ONE 2012, 7, e32977. [CrossRef]

42. Li, P.; Tai, C.; Deng, Z.X.; Gan, J.H.; Oggioni, M.R.; Ou, H.Y. Identification and characterization of chromosomal relBE toxin-antitoxin locus in Streptomyces cattleya DSM46488. Sci. Rep. 2016, 6, 32047. [CrossRef] [PubMed]

43. Sevillano, L.; Diaz, M.; Santamaria, R.I. Development of an antibiotic marker-free platform for heterologous protein production in Streptomyces. Microb. Cell Fact. 2017, 16, 164. [CrossRef] [PubMed]

44. Sevillano, L.; Diaz, M.; Santamaria, R.I. Stable expression plasmids for Streptomyces based on a toxin-antitoxin system. Microb. Cell Fact. 2013, 12, 39. [CrossRef] [PubMed]

45. Guo, Y.; Quiroga, C.; Chen, Q.; McAnulty, M.J.; Benedik, M.J.; Wood, T.K.; Wang, X. RalR (a DNase) and RalA (a small RNA) form a type I toxin-antitoxin system in Escherichia coli. Nucleic Acids Res. 2014, 42, 6448-6462. [CrossRef] [PubMed]

46. Brielle, R.; Pinel-Marie, M.L.; Felden, B. Linking bacterial type I toxins with their actions. Curr. Opin. Microbiol. 2016, 30, 114-121. [CrossRef]

47. Christensen, S.K.; Gerdes, K. RelE toxins from Bacteria and Archaea cleave mRNAs on translating ribosomes, which are rescued by tmRNA. Mol. Microbiol. 2003, 48, 1389-1400. [CrossRef] [PubMed]

48. Arcus, V.L.; McKenzie, J.L.; Robson, J.; Cook, G.M. The PIN-domain ribonucleases and the prokaryotic VapBC toxin-antitoxin array. Protein Eng. Des. Sel. 2011, 24, 33-40. [CrossRef] [PubMed]

49. Schifano, J.M.; Cruz, J.W.; Vvedenskaya, I.O.; Edifor, R.; Ouyang, M.; Husson, R.N.; Nickels, B.E.; Woychik, N.A. tRNA is a new target for cleavage by a MazF toxin. Nucleic Acids Res. 2016, 44, 1256-1270. [CrossRef] [PubMed]

50. Tripathi, A.; Dewan, P.C.; Barua, B.; Varadarajan, R. Additional role for the ccd operon of F-plasmid as a transmissible persistence factor. Proc. Natl. Acad. Sci. USA 2012, 109, 12497-12502. [CrossRef] [PubMed]

51. Germain, E.; Castro-Roa, D.; Zenkin, N.; Gerdes, K. Molecular mechanism of bacterial persistence by HipA. Mol. Cell 2013, 52, 248-254. [CrossRef] [PubMed]

52. Baba, T.; Ara, T.; Hasegawa, M.; Takai, Y.; Okumura, Y.; Baba, M.; Datsenko, K.A.; Tomita, M.; Wanner, B.L.; Mori, H. Construction of Escherichia coli K-12 in-frame, single-gene knockout mutants: The Keio collection. Mol. Syst. Biol. 2006, 2. [CrossRef] [PubMed]

53. Ladant, D.; Ullmann, A. Bordatella pertussis adenylate cyclase: A toxin with multiple talents. Trends Microbiol. 1999, 7, 172-176. [CrossRef]

54. Kitagawa, M.; Ara, T.; Arifuzzaman, M.; Ioka-Nakamichi, T.; Inamoto, E.; Toyonaga, H.; Mori, H. Complete set of ORF clones of Escherichia coli ASKA library (a complete set of E. coli K-12 ORF archive): Unique resources for biological research. DNA Res. 2005, 12, 291-299. [CrossRef] [PubMed]

55. Fu, H.; Jin, M.; Ju, L.; Mao, Y.; Gao, H. Evidence for function overlapping of CymA and the cytochrome $b c_{1}$ complex in the Shewanella oneidensis nitrate and nitrite respiration. Environ. Microbiol. 2014, 16, 3181-3195. [CrossRef] [PubMed]

56. Karimova, G.; Pidoux, J.; Ullmann, A.; Ladant, D. A bacterial two-hybrid system based on a reconstituted signal transduction pathway. Proc. Natl. Acad. Sci. USA 1998, 95, 5752-5756. [CrossRef] [PubMed]

(C) 2019 by the authors. Licensee MDPI, Basel, Switzerland. This article is an open access article distributed under the terms and conditions of the Creative Commons Attribution (CC BY) license (http://creativecommons.org/licenses/by/4.0/). 